\title{
EVERY DIRECTED GRAPH HAS A SEMI-KERNEL
}

V. Chvátal, Stanford University

L. Lovász, Vanderhilt University

In a directed graph, the distance $d(u, v)$ from a vertex $u$ to a vertex $v$ is the number of edges in the shortest directed path from $u$ to $v$. It is we11-known that every tournament has a vertex $u$ such that $\mathrm{d}(\mathrm{u}, \mathrm{v}) \leq 2$ for $a 11 \mathrm{v}$; in fact, any vertex of largest outdegree is such a vertex [2]. This generalizes as follows :

Theorem : In a directed graph G, there is always a set $S$ of vertices such that

(i) $\mathrm{d}(\mathrm{u}, \mathrm{v}) \geq 2$ whenever $\mathrm{u}, \mathrm{v} \in \mathrm{S}$ and $\mathrm{u} \neq \mathrm{v}$, (ii) given any $v \notin S$ there is an $u \in S$ with $d(u, v) \leq 2$.

Proof. By induction on the number of vertices of $G$. Let $w$ be a vertex of $G$; let $G^{\prime}$ be the subgraph of $G$ induced by $\{u: d(w, u) \geq 2\}$. By the induction hypothesis, there is a set $S^{\prime}$ which works for $G^{\prime}$. If $\mathrm{d}(u, w) \leq 1$ for some $u \in S^{\prime}$, we set $S=S^{\prime}$; otherwise we set $S=S^{\prime} U\{w\}$. Obviously, $S$ has the required properties.

REMARK. A set $S$ satisfying (i) and such that

(iii) given any $v \notin S$ there is an $u \in S$ with $d(v, u) \leq 1$ is called a kernel (cf. [1]). Not every directed graph has a kernel.

\section{REFERENCES}

1. C. Berge, Graphs and Hypergraphs, North Holland, Amsterdam 1973, Chapter 14. Kernels and Grundy functions.

2. H.G. Landau, On dominance relations and the structure of animal societies, III; the condition for a score structure, Bul1. Math. Biophys. 15 (1955), 143-148. 